\title{
PRODUTIVIDADE DA CASTANHA DE CAJU NO ESTADO DO CEARÁ: UMA ANÁLISE DE CONVERGÊNCIA
}

\author{
Otoniel Rodrigues dos Anjos Junior ${ }^{1}$ \\ Juliane da Silva Ciríaco ${ }^{2}$ \\ Márcia Cristina Silva Paixão ${ }^{3}$
}

\begin{abstract}
RESUMO
O artigo analisa a convergência de produtividade da castanha de caju no Ceará. Para tanto, utilizam-se instrumentos da econometria espacial porque a hipótese de dados espacialmente dependentes não pode ser rejeitada. A partir dos resultados significativos do I de Moran e do LISA, destacam-se evidências de que municípios com alta produtividade estão geralmente localizados próximos de outros de alta produtividade. Por sua vez, municípios de baixa produtividade, geralmente situam-se na vizinhança de outros de baixa produtividade. Os dados também mostram que a hipótese de convergência não pode ser rejeitada em nenhum dos períodos analisados. Em tais condições, estimou-se as velocidades de convergência de $6,49 \%, 4,96 \%$ e $7,71 \%$ ao ano para os períodos $2000-2007,2008-2015$ e 2000 2015, respectivamente. Por fim, considerando o período 2000-2007, identificou-se que seriam necessários cerca de 13 anos para eliminar $50 \%$ da diferença de produtividade que separa esse setor de seu estado estacionário. No caso dos períodos 2008-2015 e 2000-2015, seriam necessários cerca de 16 e 14 anos, respectivamente.
\end{abstract}

Palavras-chave: convergência de produtividade, econometria espacial, setor cajucultor.

\section{PRODUCTIVITY OF CASHEW NUT IN THE CEARÁ STATE: A CONVERGENCE ANALYSIS}

\begin{abstract}
This paper analyzes the productivity convergence in the cashew nut sector of the Ceará State, Brazil. Instruments of spatial econometrics are applied since the hypothesis of spatial dependence cannot be rejected. From the significant results of Moran's I and LISA, we highlight that municipalities with high productivity are generally located close to others with high productivity. In turn, low-productivity municipalities are generally near low-productivity ones. The data also show that the convergence hypothesis cannot be rejected in any analyzed period. Under these

\footnotetext{
${ }^{1}$ Graduado em Economia (UFPB). Mestre em Economia (PPGE-UFPB). E-mail: pbdosanjos@hotmail.com. ${ }^{2}$ Graduada em Economia (UFC). Mestra em Economia (PPGE-UFPB). E-mail: julianeciriaco@hotmail.com.

${ }^{3}$ Graduada em Economia (UFPB). Mestra em Economia (PPGE-UFPB). Doutora em Economia (UNB). Professora do Departamento de Economia da Universidade Federal da Paraíba (DE-UFPB). E-mail: marciapaixão@terra.com.br.
} 
conditions, convergence rates of $6.49 \%, 4.96 \%$ and $7.71 \%$ per year were estimated for the periods 2000-2007, 2008-2015 and 2000-2015, respectively. Finally, considering the period 2000-2007, it was identified that it would take about 13 years to eliminate $50 \%$ of the productivity gap separating this sector from its steady state. Regarding the periods 2008-2015 and 2000-2015, it would be necessary about 16 and 14 years, respectively.

Keywords: cashew nut sector, productivity convergence, spatial econometrics.

\section{INTRODUÇÃO}

O Brasil, devido sua extensão territorial, tem no setor agrícola uma relevante fonte de geração de emprego e renda. Entre as principais características desse setor, destacam-se a capacidade de fornecimento de matérias primas para os demais setores da economia e de geração de expressivos excedentes para exportação. Ainda, a produção agrícola brasileira contribui para o crescimento econômico do país ao criar postos de trabalho e garantir o provimento de alimentos para o campo e para a cidade.

O setor agrícola brasileiro é caracterizado por uma diversidade de tamanho das propriedades rurais, envolvendo desde unidades familiares até latifúndios, além de diferenças tecnológicas, regionais e climáticas que, naturalmente, influenciam a quantidade e a qualidade dos produtos.

Ao mesmo tempo em que a extensão de terras do Brasil favorece uma elevada produção agrícola, dificulta a execução de políticas públicas setoriais, sobretudo devido a diversidades regionais existentes. Isto é, tem-se em um mesmo país regiões com setores agrícolas bem diferentes em que políticas homogêneas podem, inclusive, reduzir os ganhos de diversos segmentos do setor.

Antes de qualquer decisão voltada à agricultura, é essencial entender os desdobramentos que poderão ocorrer por todo o sistema atingido. Medidas comuns para diferentes regiões podem não surtir o efeito desejado e acabar piorando globalmente os resultados. Salienta-se que tais cuidados devem ser tomados até mesmo dentro de espaços menores como estados e municípios.

O setor agrícola também apresenta características peculiares como o tipo de lavoura cultivada (temporária ou permanente), a técnica de manejo, a adaptabilidade da plantação ao clima, a tecnologia de produção, entre outras. Essa diferenciação faz surgir regiões que podem oferecer melhores condições de cultivo para algumas lavouras comparativamente a outras.

No Brasil, a cadeia produtiva do caju é atividade típica da Região Nordeste que concentra o cultivo, a extração e o processamento (MOURA; MAGALHÃES, 2008; GUANZIROLI et al., 2010). Segundo Anjos Junior et al. (2016), esse fruto se adapta justamente às condições climáticas dessa Região que, por sua vez, já possui maneiras mais apropriadas para lidar com a tecnologia de produção e o beneficiamento do produto.

Dados do Instituto Brasileiro de Geografia e Estatística (IBGE) mostram que a Região Nordeste responde por nada menos que $99,39 \%$ da área colhida de castanha de caju do país. Os dados de quantidade produzida também comprovam que a Região tem o monopólio e responde por $98,14 \%$ da produção brasileira. ${ }^{4}$

\footnotetext{
${ }^{4}$ Área colhida (em hectare): Nordeste (582.926), Norte (2.945), Centro-Oeste (652). Quantidade produzida (em tonelada): Nordeste (100.578), Norte (1.651), Centro-Oeste (256). (IBGE, 2015).
} 
Destaque-se que essa produção não se dá de forma homogênea dentro da Região Nordeste. Dados do IBGE (2015) mostram que o estado do Ceará apresenta a maior proporção de área colhida $(64,23 \%)$, seguido pelo Piauí $(14,99 \%)$ e o Rio Grande do Norte $(13,51 \%)$. Ou seja, os três maiores produtores responderam por 92,73\% de toda a área colhida de castanha de caju nordestina em 2015.

Em termos de quantidade produzida (em toneladas), os dados mostram a seguinte participação desses mesmos estados no ano de 2015: Ceará, 51,82\%; Rio Grande do Norte, 22,21\%; Piauí, 12,67\%; Bahia, 4,52\%. ${ }^{5}$

Destaque-se também que a castanha de caju responde por parcela significativa da balança comercial do estado do Ceará. Segundo Anjos Junior et al. (2016), entre os anos de 2007 e 2014, as exportações cearenses representaram cerca de $80 \%$ do volume brasileiro de exportações de castanha de caju. Assim sendo, o setor impulsiona o crescimento econômico de pequenos municípios cearenses. Segundo Moura e Magalhães (2008), os ganhos mais recentes no setor têm proporcionado alcançar o patamar de atividade agrícola demandante de tecnologia.

Diante do exposto, e considerando a importância do setor cajucultor para geração e manutenção de renda no estado do Ceará, este estudo analisa o processo de convergência da produtividade em seus municípios. Especificamente, pretende-se expor a possível diferenciação na velocidade de convergência da produtividade do setor produtor de castanha de caju no Ceará. São avaliados dados de três períodos: 2000- 2007, 2008-2015 e 2000-2015.

O artigo está dividido em quatro seções, além desta introdução e das considerações finais. Na segunda seção são contemplados, brevemente, os aspectos gerais da análise de convergência e sua aplicação à agropecuária brasileira. A terceira seção descreve a metodologia e o banco de dados utilizado. $\mathrm{Na}$ quarta, são apresentados e discutidos os principais resultados do estudo.

\section{ANÁLISE DE CONVERGÊNCIA APLICADA À AGROPECUÁRIA NO BRASIL}

Almeida, Perobelli e Ferreira (2008) investigaram a convergência espacial da produtividade agrícola nas microrregiões brasileiras entre os anos de 1991 e 2003. Concluíram que a importância da agricultura para o crescimento econômico tende a ser desigual para as diferentes regiões do país e que existem evidências em favor da convergência da produtividade.

Segundo Lopes (2004), a convergência é um processo em que uma mesma variável apresenta diferentes valores para diferentes regiões (municípios, estados, países) e que, ao longo dos anos, essa diferença se reduz. Nessa ótica, estudos mostram, por exemplo, que a convergência de renda pode ser entendida como a tendência para uma redução das diferenças de rendimentos em nível mundial (FAGERBERG; GODINHO, 2005).

Segundo Barreto e Almeida (2009), há relação positiva entre investimento em pesquisa, crescimento econômico e convergência de renda no setor agrícola. Enquanto Castro, Almeida e Lima (2015) constataram que a disparidade regional existente na produtividade da soja tende a reduzir no longo prazo.

\footnotetext{
${ }^{5}$ Sergipe não apresenta dados de produção de castanha de caju. Em 2015, os demais estados nordestinos responderam pela seguinte parcela de área colhida: Bahia, 3,67\%; Maranhão, 2,05\%; Paraíba, 0,69\%; Pernambuco, 0,65\%; Alagoas, $0,20 \%$. E em termos de quantidade produzida dos demais estados, tem-se: Maranhão, 4,07\%; Pernambuco, 3,15\%; Paraíba, 0,95\%; Alagoas, 0,1\%.
} 
Freitas e Almeida (2015), por sua vez, testam a hipótese de convergência de renda para 148 países no período de 1985 a 2005 e encontram resultados em consonância com a convergência em clubes ou múltiplos equilíbrios (QUAH, 1996).

Baumol (1986) empregou a Equação 1 para analisar um suposto processo de convergência de renda para 16 países industrializados no período de 1870 a 1979:

$\frac{1}{T}\left[\ln \left(\frac{y}{n}\right)_{i, t}-\ln \left(\frac{y}{n}\right)_{i, t-1}\right]=\alpha+\beta \ln \left(\frac{y}{n}\right)_{i, t-1}+\varepsilon_{i}$

(1)

Na equação, T é o tempo, $\ln \left(\frac{y}{n}\right)$ é o logaritmo da renda per capita, $\varepsilon$ é o termo de erro e $i$ é o indexador para os diversos países. Se existir convergência absoluta, o $\beta$ deverá apresentar sinal negativo e significativo. A convergência de renda tem implicações relevantes sobre o processo de crescimento e desenvolvimento de diferentes regiões e a relação econômica entre elas, bem como sobre a atuação das políticas públicas nesse processo (FREITAS; ALMEIDA, 2015).

Lopes (2004) analisando a produtividade da terra, aponta três razões que pode explicar a convergência da produtividade agrícola: a) ocorrência de mudanças estruturais no processo de produção (relacionadas a inovações tecnológicas e ações de políticas públicas); b) influência do mecanismo de difusão tecnológico de novas sementes, novos sistemas de plantio; c) eliminação de obstáculos ao crescimento da produção (restrições ambientais, falta de logística adequada e carência de financiamento).

Existem ainda outras formulações matemáticas capazes de auxiliar a tomada de decisão econômica considerando a ação de convergência. Nesse caso, a partir da estimação do $\beta$ é possível calcular tanto a velocidade de convergência $(\theta)$ quanto a meia-vida ( $\mathrm{T}$ ), definidas pelas equações (2) e (3), respectivamente:

$\theta=\frac{\ln (1+T \beta)}{T}$

em que T é o tempo.

$\tau=\frac{\ln (2)}{\ln (1+\beta)}$

(3)

Salienta-se que meia-vida $(\mathrm{T})$ pode ser entendido como o tempo necessário para que as economias percorram metade do caminho que as separam de seus estados estacionários.

\section{METODOLOGIA E BANCO DE DADOS}

Esta seção discute os procedimentos metodológicos empregados neste estudo. Inicialmente, são apresentados aspectos gerais da análise exploratória empreendida, apontando testes e procedimentos que mostram como identificar existência de padrão espacial nos dados. Em seguida, discute-se sucintamente a 
estratégia empírica adotada para a estimação de modelos econométricos incorporando a ação da dependência espacial. Por fim, apresentam-se informações sobre os dados utilizados.

\subsection{ANÁLISE EXPLORATÓRIA DE DADOS ESPACIAIS}

$\mathrm{Na}$ ótica de Almeida (2012), a modelagem espacial tem como objetivo especificar, estimar, testar e prever modelos teóricos influenciados por efeitos espaciais. Tal modelagem difere da tradicional por incorporá-lo tanto na estimação quanto no teste de hipótese e previsão dos modelos. Já Anselin (1988, 2003) argumenta que a modelagem espacial apresenta qualidade que possibilita estudar fenômenos com variáveis espacialmente intensivas. Assim, os instrumentos econométricos dessa modelagem acabam respondendo eficientemente aos tipos de dados ou problemas encontrados no setor agropecuário brasileiro.

A Análise Exploratória de Dados Espaciais (AEDE) deve ser tarefa preliminar a qualquer análise estatística mais sofisticada (ANSELIN, 1999; BRUNSDON, FOTHERINGHAM; CHARLTON, 2002; ALMEIDA, 2012). Na perspectiva de Anselin (1999), a AEDE permite descrever e visualizar padrões espaciais, identificar localidades atípicas (outliers espaciais) e descobrir padrões de associação espacial (clusters espaciais).

Almeida (2012) aponta tal análise como a mais indicada na investigação de variáveis densas ou intensivas. As variáveis extensivas podem conduzir a resultados enganosos devido à possibilidade de tendência de correlação espúria com a área ou população total da região em análise (ANSELIN, 2005).

Moran (1948) elaborou um indicador de autocorrelação espacial capaz de realizar consistentemente a AEDE. A estatística I de Moran pode ser utilizada na detecção da autocorrelação espacial global. Tal indicador pode ser representado por meio de um valor único representando o grau de associação espacial presente nos dados. Formalmente, o I de Moran Global pode ser expresso da seguinte forma:

$\boldsymbol{I}=\frac{N}{\sum_{i} \sum_{j} w_{i j}} \frac{\sum_{i} \sum_{j} w_{i j}\left(y_{i t}-\bar{y}_{t}\right)\left(y_{j t}-\bar{y}_{t}\right)}{\sum_{i}\left(y_{i t}-\bar{y}_{t}\right)^{2}}$

$N$ é o número de regiões, $w_{i j}$ são os elementos da matriz de pesos espaciais $(\boldsymbol{W}), y_{i t}$ é a observação na região $i$ no período $t$ e $\bar{y}_{t}$ é a média das observações entre as regiões no período $t$.

Conforme Anselin (1995), a matriz $\boldsymbol{W}$ contempla as informações referentes à dependência espacial entre as $N$ regiões $i$. Os elementos $w_{i j}$ indicam a associação espacial entre as regiões $i$ e $j$, sendo os elementos na diagonal principal iguais a zero. Assim, tem-se que a estatística de Moran assume valores entre um (1) e menos um (-1) e possui valor esperado igual:

$E[I]=-[1 /(n-1)]$

(5) 
Da equação (5) têm-se duas informações bastante relevantes. Inicialmente, nota-se que essa equação representa o valor obtido quando não há padrão espacial nos dados. Secundariamente, nota-se que tal estatística se aproxima de zero quando o número de municípios aumenta $(n \rightarrow \infty)$. Portanto, valores positivos de $I$ que excedem $E[I]$ indicam autocorrelação espacial positiva, enquanto valores de $I$ abaixo do $E[I]$ sinalizam autocorrelação espacial negativa.

Apesar da eficiência em mapear globalmente a incidência de autocorrelação espacial, a utilização do teste de Moran global pode deixar de captar padrões espaciais locais significantes (clusters e outliers) em algumas regiões (ANSELIN, 1994). Nesse sentido, Anselin (1995) aponta que é possível encontrar um teste de associação espacial local a partir de fragmentos do teste de Moran global.

A análise da autocorrelação espacial local pode ser efetuada utilizando-se o I de Moran Local ou LISA (sigla de Local Indicator of Spatial Association). Segundo Anselin (1995), esse indicador fornece indicação de clusters ou agrupamentos espaciais significativos de valores semelhantes em torno de certa observação. $O$ LISA para cada região $i$ e período $t$ pode ser expresso por:

$\boldsymbol{I}_{i}=\frac{\left(y_{i t}-\bar{y}_{t}\right) \sum_{j} w_{i j}\left(y_{j t}-\bar{y}_{t}\right)}{m_{0}}$

(6)

Em que $m_{0}=\frac{\sum_{i}\left(y_{i t}-\bar{y}_{t}\right)^{2}}{N}$.

Valores positivos de $\boldsymbol{I}_{i}$ devem indicar presença de agrupamentos de valores similares entre si e valores negativos, presença de agrupamentos de valores relativamente dissimilares.

\subsection{MODELANDO A DEPENDÊNCIA ESPACIAL}

Na presença de efeitos espaciais, o Modelo Clássico de Regressão Linear (MCRL) apresenta limitações que desencadeiam inconsistência e/ou ineficiência (ANSELIN, 1988; ANSELIN; BERA, 1998). Nessa perspectiva, a estimação do MCRL no contexto de dependência espacial e averiguando a produtividade média (Equação 7), objetiva apenas encontrar como a dependência espacial toma forma no espaço (na variável dependente e/ou no termo de erro). A forma da dependência espacial pode ser constatada por meio dos testes do Multiplicador de Lagrange e Multiplicador de Lagrange Robusto. ${ }^{6}$

$\ln \left(\frac{P m e_{t}}{P m e_{t-n}}\right)=\alpha+\beta \ln \left(P m e_{t-n}\right)+u_{i}$

(7)

\footnotetext{
${ }^{6}$ Para mais informações, consultar Florax, Folmer e Rey (2003).
} 
Sendo que $\ln \left(\frac{P m e_{t}}{P m e_{t-n}}\right)$ é o logaritmo natural da razão entre a produtividade média entre dois anos, $\ln \left(P m e_{t-n}\right)$ é o logaritmo natural da produtividade média no período inicial e $u_{i}$ é o termo de erro. Ao passo que o termo de erro $u_{i}$ segue um processo espacial autorregressivo, tem-se:

$$
u_{i}=\lambda u_{i}+\varepsilon_{i}
$$

(8)

Em que $\lambda$ representa o coeficiente escalar do erro espacial e o termo de erro $\mathcal{E}_{i}$ é normalmente distribuído com média zero e variância constante. Incorporando a Equação (8) na Equação (7), encontra-se a forma adequada do modelo de regressão de erro espacial:

$\ln \left(\frac{P m e_{t}}{P m e_{t-n}}\right)=\alpha+\beta \ln \left(P m e_{t-n}\right)+(I-\lambda W) \varepsilon_{i}$

(9)

Nessa modelagem, $\boldsymbol{W}$ é a mesma matriz de contiguidade utilizada para realizar AEDE. Com $\lambda \neq 0$, a incidência de determinado choque numa região se espalha não só para os seus vizinhos imediatos, mas afeta também os vizinhos de ordem maior (REY; MONTOURI, 1999). Assim, o coeficiente espacial autorregressivo $(\lambda)$ mede o grau de dependência espacial no termo de erro ou de efeitos não modelados que não são aleatoriamente distribuídos através do espaço.

Outra forma de internalizar os efeitos dos transbordamentos é através da modelagem global de defasagem espacial. Nesse caso, mudanças na variável explicativa numa região afetarão diretamente a própria região e poderão afetar as demais regiões por meio de efeito indireto (LESAGE; PACE, 2009).

$\ln \left(\frac{P_{m e_{t}}}{P_{m e_{t-n}}}\right)=\alpha+\rho W \ln \left(\frac{P_{m e_{t}}}{P_{m e_{t-n}}}\right)+\beta \ln \left(P m e_{t-n}\right)+\tau W \ln \left(P m e_{t-n}\right)+\xi$

Em que $\xi=\lambda W \xi+\mathcal{E}_{i}$ é o termo de erro; $\ln \left(\frac{P m e_{t}}{P m e_{t-n}}\right)$ é o logaritmo natural da razão entre produtividade média de dois anos. Por sua vez, $\ln \left(P m e_{t-n}\right)$ é o logaritmo natural da produtividade média no período inicial; т é o termo de transbordamento espacial, enquanto $W \ln \left(\frac{P m e_{t}}{P m e_{t-n}}\right)$ denota a defasagem espacial da produtividade e $\mathcal{E}$ representa o termo de erro com média zero e variância constante. Por fim, $\rho$ é o coeficiente de defasagem o qual espera-se 
que seja maior do que zero sugerindo, então, existência de autocorrelação espacial positiva.

Por fim, tem-se a Equação (11) representando uma junção das equações (9) e (10) em que os transbordamentos podem ocorrer tanto na variável dependente quanto no termo de erro do modelo.

$\ln \left(\frac{P_{m \theta_{t}}}{P_{m \theta_{t-n}}}\right)=\alpha+\rho W_{1} \ln \left(\frac{P_{m \theta_{t}}}{P_{m \theta_{t-n}}}\right)+\beta \ln \left(P m e_{t-n}\right)+\tau W \ln \left(P m e_{t-n}\right)+\lambda W_{2} \xi+\varepsilon_{i}$ (11)

em que $\boldsymbol{W}_{1}$ e $\boldsymbol{W}_{2}$ são matrizes de contiguidade não necessariamente iguais.

\subsection{DADOS DA PESQUISA}

São utilizados dados de rendimento médio por hectare da castanha de caju no estado do Ceará. Tais dados representam a produtividade do setor e são levantados pela Pesquisa Agrícola Municipal (PAM) do Instituto Brasileiro de Geografia e Estatística (IBGE) dos anos 2000, 2007, 2008 e 2015.

Nesta pesquisa, utiliza-se o rendimento médio municipal do fruto como forma de verificar um possível processo de convergência setorial de renda. As estimações de modelos de convergência absoluta testam o processo sem considerar o efeito de outras variáveis relacionadas ao fenômeno.

Destaque-se que a presente pesquisa enfrentou limitações de bases de dados adequadas para explicar o rendimento médio do setor cajucultor no Ceará. A falta de informações sobre fatores naturais (solo e clima), estruturais e tecnológicos (tanques, lagos, açudes, construções, benfeitoria e sistema de plantio), humano (mão de obra e nível de especialização), organizacional (nível de organização do setor em cada município), existência de cooperativas e/ou associação de moradores e de mercado específico para a venda e escoamento do produto, limitaram essa análise a um modelo de convergência absoluta.

Nesse sentido, a inclusão de controles em um possível modelo de convergência relativa poderá ser efetuada em pesquisas futuras as quais já partam da condição de convergência absoluta.

\section{RESULTADOS}

Nesta seção são descritos os resultados da pesquisa. Inicialmente, as estatísticas descritivas e os resultados da análise exploratória de dados espaciais são apresentados e, em seguida, discutem-se os principais resultados das estimações dos modelos econométricos.

\subsection{A PRODUÇÃO DE CASTANHA DE CAJU NO ESTADO DO CEARÁ: FATOS ESTILIZADOS}

$\mathrm{Na}$ Figura 1 tem-se a produção total dos três maiores produtores de castanha de caju do Nordeste nos anos de 2000, 2005, 2010 e 2015. Com destaque o estado Ceará no ano de 2005 com mais de 66 mil toneladas. No período 2000-

\footnotetext{
${ }^{7}$ Dados disponíveis em: <https://sidra.ibge.gov.br/tabela/106>. Acesso em: dez. 2016.
} 
2015, sua produção média foi 77.161 toneladas quase o dobro do Rio Grande do Norte, segundo colocado com média de 33.739 toneladas.

Figura 1 - Estados da Região Nordeste com maior produção de castanha de caju nos anos de 2000, 2005, 2010 e 2015 (toneladas)

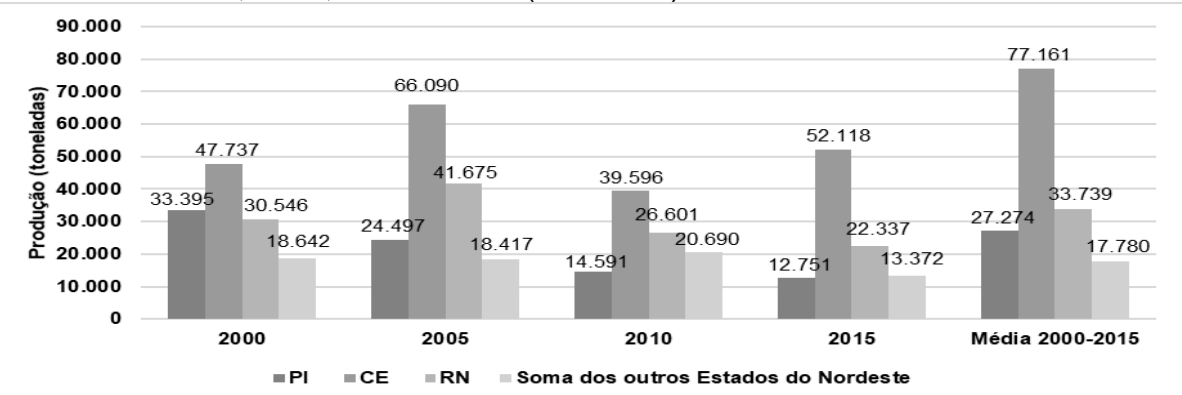

Fonte: Elaboração dos autores a partir dos dados da Produção Agrícola Municipal do IBGE.

Por fim, aparece o estado do Piauí que, exceto para o ano 2000, é o terceiro produtor do Nordeste. Portanto, os três maiores produtores de castanha de caju concentram mais de $75 \%$ da produção da Região em qualquer dos anos analisados.

Aparentemente, o fruto contribui com a geração de emprego e renda no Ceará, comercializado nos mercados interno e externo. Considerando apenas o período 2000-2015, o estado respondeu por 70-80\% das exportações nordestinas do fruto (Figura 2).

Figura 2 - Participação das exportações cearenses nas exportações nordestinas de castanha de caju no período $2000-2015$

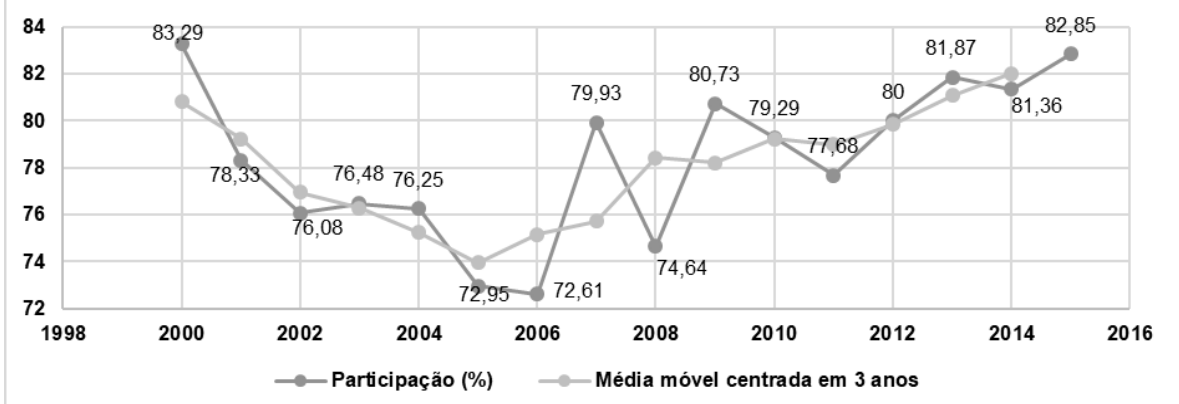

Fonte: Elaboração dos autores a partir dos dados da Produção Agrícola Municipal do IBGE.

Elaborou-se um sistema de média móvel centrada em três anos para melhor demonstrar a tendência das exportações ao longo do tempo (Figura 2), possibilitando visualizar uma parábola convexa em relação a origem. Portanto, a série começa com valores expressivamente altos e passa a apresentar reduções ao longo dos anos atingindo um mínimo global no ano de 2005 e, posteriormente, crescendo monotonamente até o último ano da série.

Ressalte-se que os períodos de queda das exportações cearenses são avaliados na literatura como de perda de vantagens comparativas de suas 
exportações em relação aos estados do Rio Grande do Norte e Piauí e de outros países como Vietnã e Índia (ALBUQUERQUE et al., 2011).

Os dados apresentados na Figura 3 sugerem perca de relevância das exportações de castanha de caju na balança comercial cearense. Um fenômeno que pode ser reflexo de uma série de fatores: surgimento de mercados ofertantes, mudança de qualidade do fruto, aumento do investimento em outros setores da economia e consequente ascendência de novos produtos na pauta de exportações do estado.

Figura 3 - Participação das exportações de castanha de caju na balança comercial cearense entre 2000 e 2015

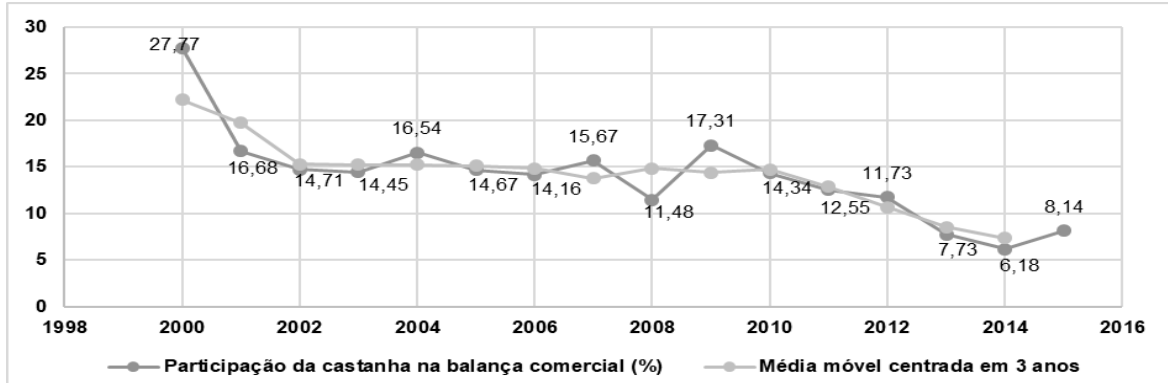

Fonte: Elaboração dos autores a partir dos dados da Produção Agrícola Municipal do IBGE.

\subsection{ESTATÍSTICA DESCRITIVA DOS DADOS}

$\mathrm{Na}$ Tabela 1, constata-se que a produção de castanha está sujeita a um grau relevante de oscilação. Esse comportamento pode ser reflexo de fatores como variações climáticas, tecnológicas ou mesmo falta de incentivos ao produtor.

Segundo Anjos Junior et al. (2016), a redução no rendimento da castanha de caju pode ser ocasionada por: baixa fertilidade da terra, chuvas escassas, pouco investimento no setor, ausência de especialização no processo de produção, entre outros.

Tabela 1 - Estatística descritiva da produtividade média da castanha de caju por hectare no estado do Ceará nos anos de 2000, 2008 e 2015

\begin{tabular}{lrrr}
\hline \multicolumn{1}{c}{ Estatística } & $\mathbf{2 0 0 0}$ & $\mathbf{2 0 0 8}$ & \multicolumn{1}{c}{$\mathbf{2 0 1 5}$} \\
\hline Média & 222,77 & 347,17 & 123,44 \\
Erro padrão & 8,93 & 9,63 & 7,31 \\
Mediana & 200 & 347 & 110 \\
Desvio padrão & 121,13 & 130,57 & 99,22 \\
Mínimo & 0 & 0 & 0 \\
Máximo & 500 & 1000 & 500 \\
Contagem & 184 & 184 & 184 \\
Nível de confiança (95\%) & 17,62 & 18,99 & 14,43 \\
\hline
\end{tabular}

Fonte: elaboração dos autores a partir dos dados da pesquisa.

$\mathrm{Na}$ comparação das estatísticas descritivas para os anos 2000, 2008 e 2015, tem-se que os valores de 2015 sugerem ser estatisticamente mais robustos comparativamente aos demais. No entanto, tais informações não permitem tirar conclusões precisas, sobretudo pelas oscilações apresentadas em seus indicadores. Assim, faz-se necessária a estimação de modelos econométricos específicos que permitam obter uma conclusão mais consistente acerca do fenômeno estudado. 


\subsection{DEPENDÊNCIA ESPACIAL DA PRODUTIVIDADE DA CASTANHA DE CAJU NOS MUNICÍPIOS CEARENSES}

Almeida (2012) aponta o I de Moran como um eficiente indicador para ser empregado no processo de identificação de incidência de autocorrelação espacial. O autor também destaca a existência de outros testes que podem levar a resultados similares, como os testes de Geary (1954) ou Getis e Ord (1992).

Outro aspecto relevante é o procedimento preliminar necessário à realização de testes de dependência e estimação de modelos econométricos que é o de encontrar determinada matriz que possibilite capturar o máximo de associação espacial possível. Para tanto, utiliza-se a matriz binária de pesos espaciais $(\boldsymbol{W})$ que atribui valor 1 (um) para o caso em que dois municípios são vizinhos e valor 0 (zero) no caso contrário. No mais, essa matriz de vizinhança do tipo Queen de primeira ordem é normalizada na linha.

$\mathrm{Na}$ Tabela 2, mostram-se os resultados do / de Moran, de seu valor esperado, o desvio padrão e o z-value para a produtividade média da castanha de caju nos municípios cearenses nos anos de 2000, 2008 e 2015. Esses resultados foram obtidos considerando $99 \%$ de confiança e 999 permutações aleatórias. Logo, rejeita-se a $1 \%$ de significância, a hipótese nula de aleatoriedade. Em outros termos, com valores positivos de $I$ excedendo $E[I]$, sugere-se ocorrência de dependência espacial positiva nos anos selecionados.

Tabela 2 - I de Moran, I de Moran esperado, desvio padrão e z-value para produtividade média da castanha de caju nos municípios cearenses para os anos de 2000, 2008 e 2015

\begin{tabular}{cccccc}
\hline Ano & $I$ de Moran & $E[I]$ & Média & Desvio Padrão & $z$ - value \\
\hline 2000 & 0,396 & $-0,005$ & $-0,004$ & 0,047 & 8,545 \\
2008 & 0,352 & $-0,005$ & $-0,006$ & 0,046 & 7,834 \\
2015 & 0,233 & $-0,005$ & $-0,004$ & 0,046 & 5,164 \\
\hline
\end{tabular}

Fonte: elaboração dos autores a partir dos dados da pesquisa.

A partir da Tabela 2, constata-se que o indicador de autocorrelação espacial global se reduz no tempo. No período $2000-2008$, o valor diminuiu $11,11 \%$ e no período 2008-2015, 33,81\%; por fim, considerando todo o período entre 2000 e 2015 , esse indicador contrai $41,16 \%$. Pode-se afirmar, portanto, que a produtividade da castanha de caju de determinado município depende não apenas de seu resultado, mas também do resultado dos municípios que formam sua vizinhança e que essa dependência se reduz ao longo do tempo.

Ainda, o valor positivo do $l$ de Moran sugere que municípios de alta produtividade média estão mais próximos daqueles municípios que também apresentam alta produtividade média. Contrariamente, municípios que possuem baixa produtividade média estão associados a municípios com essa mesma característica.

Para qualificar os resultados obtidos, também pode-se elaborar os diagramas de dispersão de Moran global e o indicador local ou LISA. A interpretação de tais estatísticas é relativamente simples, pois o indicador global é representado por um gráfico temático composto por todas as ocorrências da variável dependente e dividido em quatro quadrantes. ${ }^{8}$ Por sua vez, o LISA é formado por um mapa

\footnotetext{
${ }^{8}$ Q1 (Alto-Alto, AA), Q2 (Alto-Baixo, AB), Q3 (Baixo-Baixo, BB) e Q4 (Baixo-Alto, BA).
} 
temático composto por cinco cores distintas, cada qual representando um tipo de associação espacial.

Anselin (1996) aponta o diagrama de dispersão de Moran como uma das formas de interpretar a estatística I de Moran uma vez que é uma representação do coeficiente ou inclinação da reta de regressão e possibilita visualizar a correlação linear entre duas variáveis. Na presente avaliação, tem-se no eixo horizontal a produtividade média para os anos selecionados de 2000, 2008 e 2015 (R00, R08, R15), enquanto apresenta-se no eixo vertical a defasagem espacial da variável de interesse para o mesmo período (W R00, W R08, W R15), ver Quadro 1.

Inicialmente, verifica-se a composição do indicador de Moran global, constata-se no primeiro coorte, referente ao ano 2000 , que: $28,80 \%$ dos municípios apresentavam comportamento do tipo Alto-Alto (AA); $41,85 \%$ do tipo Baixo-Baixo (BB); $17,39 \%$ do tipo Baixo-Alto (BA); e 11,96\% do tipo Alto-Baixo (AB). Já no ano de 2008 , tem-se $40,22 \%$ de relação do tipo Ato-Alto e $43,48 \%$ do tipo Baixo-Baixo. Sequencialmente, as relações Baixo-Alto e Alto-Baixo apresentaram valores de $7,06 \%$ e $9,24 \%$, respectivamente.

Quadro 1 - Resumo das Estatísticas do I de Moran Global e do LISA para os anos de 2000,2008 e 2015

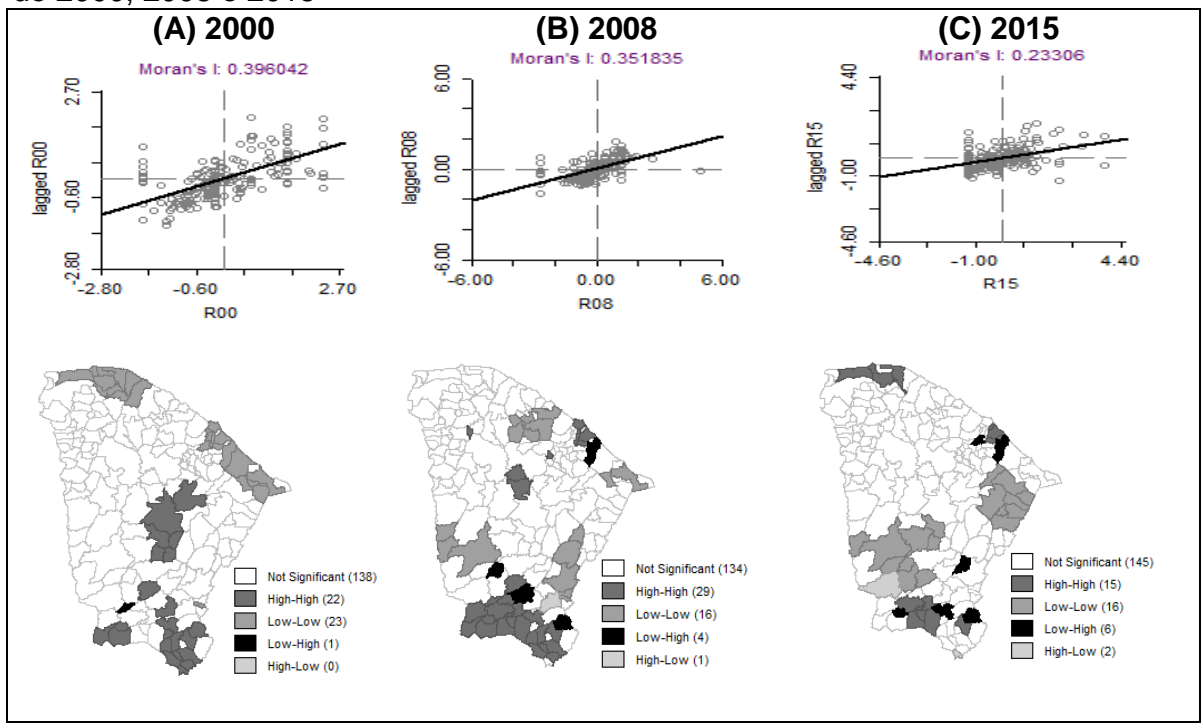

Fonte: elaboração dos autores a partir dos dados da pesquisa e utilizando o software Geoda.

Sobre o ano de 2015, 33,69\% dos municípios apresentaram relação do tipo Alto-Alto. Por sua vez, $40,76 \%$ dos municípios apresentam baixo rendimento do fruto e são circundados por outros que também apresentam baixo rendimento médio por hectare. Outros $12,5 \%$ dos municípios apresentam baixo rendimento médio e são cercados por municípios de alto rendimento médio, enquanto aqueles que apresentam alto rendimento médio e são cercados por municípios de baixo rendimento médio representam $13,04 \%$.

Há expressiva proporção de municípios que apresentam relação mais similar ( $\mathrm{AA}$ e $\mathrm{BB}$ ) comparativamente àqueles mais distintos entre si (BA e $\mathrm{AB}$ ). A reta de regressão já havia, previamente, exposto tal resultado, pois sua inclinação positiva representa justamente isso. Ao passo que se soma os casos mais similares, 
o resultado é sempre maior a $70 \%(70,65 \%$ em $2000,83,70 \%$ em 2008 e $74,45 \%$ em 2015). Consequentemente, os casos mais distintos não atingem $30 \%(29,35 \% \mathrm{em}$ $2000,16,30 \%$ em 2008 e $25,55 \%$ em 2015).

Como o indicador de associação espacial global pode falhar no processo de captura de alguns padrões locais significantes, pode-se utilizar o LISA (ANSELIN, 1995). Tal indicador nos anos de 2000, 2008 e 2015, sinaliza comportamento padronizado, ao nível de significância considerado, apenas em 24,46\%, 27,17\% e $21,20 \%$ dos municípios do Ceará respectivamente.

No ano 2000 entre os municípios significantes localmente 44,44\% Alto-Alto, $53,33 \%$ Baixo-Baixo e 2,22\% Baixo-Alto e não apresenta caso Alto-Baixo. Os dados mais similares entre si representam $97,77 \%$. Por sua vez, em 2008, tais valores são de $61,22 \%$ (AA), 30,61\% (BB), 6,67\% (BA) e 2,04\% (AB) respectivamente. Os mais similares totalizam $91,83 \%$ dos casos significantes e os mais dissimilares (BA e $A B$ ) correspondem a $8,17 \%$.

Finalmente, no ano de 2015 têm-se as seguintes proporções: 76,47\% (AA), $43,24 \%$ (BB), $13,51 \%$ (BA) e $8,11 \%$ (AB). A similaridade é de $78,37 \%$ e a dissimilaridade $21,63 \%$. Diante do exposto, há indícios para se acreditar em dados de produtividade média de castanha não aleatorizados no espaço.

\subsection{ESTIMAÇÕES DOS MODELOS ECONOMÉTRICOS}

Na Tabela 3, tem-se o diagnóstico da estimação do Modelo Clássico de Regressão Linear (MCRL). O resultado significativo encontrado na estatística de Moran aplicado nos resíduos para os anos selecionado de 2000-2007 $(I=6,9857)$, 2008-2015 ( $I=5,571)$ e 2000-2015 ( $I=6,5709)$ sugerem distribuição não aleatória de dados ao longo do território cearense e tal fato torna estéril as estimativas do MCRL. Nesse caso, modelos econométricos que incorporem os efeitos dos transbordamentos espaciais podem tratar adequadamente o fenômeno em análise. 
Tabela 3 - Estimação econométrica do MCRL para os períodos 2000-2007, 20082015 e $2000-2015$

\begin{tabular}{|c|c|c|c|}
\hline - & $2000-2007$ & 2008-2015 & 2000-2015 \\
\hline A & 0,324 & 0,046 & 0,163 \\
\hline & $(0,000)$ & $(0,599)$ & $(0,000)$ \\
\hline B & $\begin{array}{c}-0,050 \\
(0,000)\end{array}$ & $\begin{array}{c}-0,048 \\
(0,002)\end{array}$ & $\begin{array}{l}-0,046 \\
(0,000)\end{array}$ \\
\hline \multicolumn{4}{|c|}{ Diagnóstico da Regressão } \\
\hline $\mathrm{AIC}$ & $-312,497$ & $-2,837$ & $-258,300$ \\
\hline SC & $-306,068$ & 3,593 & $-251,870$ \\
\hline LIK & 158,249 & 3,419 & 131,150 \\
\hline Teste Jarque-Bera & 661,235 & 130,623 & 61,069 \\
\hline & $(0,000)$ & $(0,000)$ & $(0,000)$ \\
\hline Teste Breusch-Pagan & $\begin{array}{c}619,681 \\
(0,000)\end{array}$ & $\begin{array}{l}14,684 \\
(0,000)\end{array}$ & $\begin{array}{c}0,115 \\
(0,735)\end{array}$ \\
\hline Multicolinearidade & 7,506 & 9,795 & 7,506 \\
\hline \multicolumn{4}{|c|}{ Diagnóstico de Dependência Espacial } \\
\hline I de Moran & $\begin{array}{c}6,986 \\
(0,000)\end{array}$ & $\begin{array}{c}5,571 \\
(0,000)\end{array}$ & $\begin{array}{c}6,571 \\
(0,000)\end{array}$ \\
\hline ML $\rho$ - defasagem & $\begin{array}{l}43,855 \\
(0,000)\end{array}$ & $\begin{array}{l}26,796 \\
(0,000)\end{array}$ & $\begin{array}{l}44,868 \\
(0,000)\end{array}$ \\
\hline MLR $\rho$ - defasagem & $\begin{array}{c}1,578 \\
(0,209)\end{array}$ & $\begin{array}{c}0,246 \\
(0.619)\end{array}$ & $\begin{array}{c}4,924 \\
(0.027)\end{array}$ \\
\hline $\mathrm{ML} \lambda$ - erro & $\begin{array}{l}45,287 \\
(0,000)\end{array}$ & $\begin{array}{l}28,447 \\
(0,000)\end{array}$ & $\begin{array}{l}39,962 \\
(0,000)\end{array}$ \\
\hline MLRA - erro & $\begin{array}{c}3,010 \\
(0,083)\end{array}$ & $\begin{array}{c}1,898 \\
(0,168)\end{array}$ & $\begin{array}{c}0,018 \\
(0,893)\end{array}$ \\
\hline ML(SARMA) & $\begin{array}{l}46,865 \\
(0,000)\end{array}$ & $\begin{array}{l}28,693 \\
(0,000)\end{array}$ & $\begin{array}{l}44,887 \\
(0,000)\end{array}$ \\
\hline
\end{tabular}

Fonte: elaboração dos autores a partir dos dados da pesquisa e utilizando o Software Geoda versão 1.8.14.

Nota: os resultados entre parênteses indicam o valor das probabilidades.

O diagnóstico da regressão linear contempla informações que podem direcionar o processo de estimação dos modelos espaciais. Inicialmente, destaca-se que o valor do teste de Jarque-Bera sugerindo não normalidade dos resíduos nos três períodos. A partir dos resultados do teste de Breusch-Pagan, tem-se que apenas o período 2000-2015 não apresenta heterocedasticidade. Por fim, o valor do teste de multicolinearidade apresenta-se dentro de parâmetro estatisticamente aceitável nos períodos selecionados (valor menor que 30).

É amplamente divulgado o processo de seleção de modelos econométricos por meio dos menores critérios de informação de Akaike $(A I C)$ e Schwartz $(S C)$, assim como o maior valor da função de verossimilhança (LIK). Em econometria espacial existe a possibilidade de utilização de outros procedimentos para seleção de modelos tão eficientes quanto o anterior. Tal estratégia consiste na utilização dos testes dos Multiplicadores de Lagrange (ML) e Multiplicador Lagrange Robusto (MLR) para identificar como a dependência espacial toma forma (ALMEIDA, 2012). Sendo a especificação correta a que apresentar-se estatisticamente significante nos dois testes (ML e MLR).

No período que se estende entre 2000 e 2007 a melhor especificação possível para modelar a dependência espacial é a Spatial Error Models (SEM), pois apresenta resultado significante tanto para os testes ML quanto MLR. Por sua vez, entre os anos de 2008 e 2015 o modelo de maior robustez é o Spatial Autorregressive and Moving Average (SARMA) dada a significância dos critérios do ML e MLR. Finalmente, considerando o período que se estende entre 2000 e 2015 a 
forma mais adequada de modelar a dependência espacial é utilizar o modelo Spatial Auto Regressive (SAR).

Nos períodos 2000-2007 e 2008-2015, a modelagem SARMA aparece como opção aos demais modelos. No entanto, tal modelo será estimado apenas nos casos em que o modelo SAR ou SEM não sejam capazes de capturar toda fonte de dependência espacial a partir dos dados observados.

A maioria dos trabalhos sobre convergência de renda pressupõe, erroneamente, que os países são unidades geográficas isoladas, não considerando os efeitos espaciais (dependência e heterogeneidade) subjacentes (FREITAS; ALMEIDA, 2015). No presente caso, o processo de estimação dos modelos espaciais deverá tratar consistentemente os efeitos de transbordamentos existentes na variável dependente nos períodos selecionados: 2000 a 2007, 2008 a 2015 e 2000 a 2015.

Kelejian e Prucha (2010) afirmam que os estimadores de máxima verossimilhança podem apresentar inconsistência ou viés na presença de heterocedasticidade. Em função disso, a estimação do modelo espacial no período de 2000 a 2007 deve seguir a especificação SEM-GMM e poderá ser fundamentada no que foi proposta por Kelejian e Prucha (1999). Por fim, utiliza-se o mecanismo (KP-HET) indicado por Kelejian e Prucha (2010) para lidar com heterocedasticidade desconhecida.

Já para estimar o modelo SARMA-GMM para o período 2008-2015, podese utilizar o procedimento de Kelejian e Prucha (1998) em conjunto com o estimador KP-HET para correção da heterocedasticidade (KELEJIAN; PRUCHA, 2010).

Finalmente, para modelar a dependência espacial para 2000-2015, deve-se seguir o proposto por Anselin $(1988)^{9}$ considerando o mecanismo de correção para heterocedasticidade e autocorrelação espacial (HAC) propostos por Kelejian e Prucha (2007). Salienta-se que o HAC é um estimador não-paramétrico para a covariância espacial, sendo robusto mesmo na presença de dependência espacial e heterocedasticidade. Em tempo, destaca-se que para acomodar tal especificação é exigida determinada função kernel do tipo triangular ou de Barlett que possa auxiliar no processo de estimação.

No Apêndice $A$, apresentam-se os resultados do teste de $\sigma$-convergência ${ }^{10}$ do rendimento médio da castanha de caju para os anos 2000, 2008 e 2015. Assim, a hipótese de $\sigma$ - convergência no setor cajucultor não pode ser rejeitada apenas no caso do período 2000-2008. Como visto, a presença de $\beta$-convergência não implica $\sigma$-convergência, porém o inverso é sempre verdadeiro. $\mathrm{Na}$ Tabela 4 , são apresentados os resultados da estimação dos modelos $\beta$-convergência considerando transbordamentos espaciais para os períodos $2000-2007$; 2008-2015 e 2000-2015.

\footnotetext{
${ }^{9}$ Spatial two-stage least squares (S2SLS) - Mínimos quadrados espaciais de dois estágios.

${ }_{10}$ Teste $\sigma$-convergência (Desvio padrão/média). Coeficiente de variação que indica dispersão e quanto maior o valor maior a diferença nos dados analisados. Caso $\sigma_{t}>\sigma_{\mathrm{kt}+} 1\left(\sigma_{\mathrm{t}}-\sigma_{\mathrm{kt}+} 1<0\right.$, evidência de convergência).
} 
Tabela 4 - Estimação econométrica dos modelos espaciais para os períodos 20002007, 2008-2015 e 2000-2015

\begin{tabular}{|c|c|c|c|}
\hline & 2000-2007 & 2008-2015 & 2000-2015 \\
\hline & SEM-GMM & SARMA-GMM & SAR-GMM \\
\hline \multirow[t]{2}{*}{ A } & 0,329 & $-0,144$ & 0,161 \\
\hline & $(0,001)$ & $(0,802)$ & $(0,014)$ \\
\hline B & $\begin{array}{c}-0,051 \\
(0,005)\end{array}$ & $\begin{array}{c}-0,042 \\
(0,014)\end{array}$ & $\begin{array}{l}-0,046 \\
(0,000)\end{array}$ \\
\hline $\mathrm{P}$ & - & $\begin{array}{c}0,873 \\
(0,638)\end{array}$ & $\begin{array}{c}0,038 \\
(0,009)\end{array}$ \\
\hline$\wedge$ & $\begin{array}{c}0,135 \\
(0,001)\end{array}$ & $\begin{array}{c}0,526 \\
(0,007)\end{array}$ & - \\
\hline $\begin{array}{l}\text { Velocidade de } \\
\text { convergência }\end{array}$ & 0,0646 & 0,0496 & 0,0771 \\
\hline Meia Vida & 13,34 & 16,22 & 14,80 \\
\hline \multicolumn{4}{|c|}{ Teste de Dependência Espacial no Resíduo do Modelo Estimado } \\
\hline I de Moran & $\begin{array}{c}0,0086 \\
(0,3430)\end{array}$ & $\begin{array}{c}0,0436 \\
(0,1450)\end{array}$ & $\begin{array}{l}- \\
-\end{array}$ \\
\hline $\begin{array}{l}\text { Teste de Anselin- } \\
\text { Kelejian }\end{array}$ & - & - & $\begin{array}{c}0,497 \\
(0,4809)\end{array}$ \\
\hline
\end{tabular}

Fonte: elaboração dos autores a partir dos dados da pesquisa e utilizando o Software Geoda versão 1.8.14 e o GeodaSpace.

Nota: os valores entre parênteses são probabilidades e pseudo p-valores associados à estatística de Moran pós estimação.

Os testes realizados apontam que os modelos utilizados foram capazes de modelar consistentemente a dependência espacial existente. Nesse caso, o melhor modelo é aquele que não apresenta evidências de autocorrelação espacial em seus resíduos. Portanto, os testes de Moran realizados para os modelos SEM e SARMA, assim como o teste Anselin-Kelejian aplicado para o modelo SAR, foram não significativos, dando credibilidade a seus estimadores.

Por meio do parâmetro $\beta$ estimado pelos diferentes modelos espaciais, constata-se que todos os modelos apresentam sinal condizente com a incidência de convergência. $E$ esses resultados estão em conformidade com os de outros estudos como o de Lopes (2004), Almeida, Perobelli e Ferreira (2008), Barreto e Almeida (2009), Freitas e Almeida (2015).

Nos períodos 2000-2007, 2008-2015 e 2000-2015, verifica-se processo de convergência estatisticamente significante. Portanto, tais períodos merecem ser melhor avaliados quanto a velocidade de convergência.

Assim, para 2000-2007, 2008-2015 e 2000-2015, têm-se valores de $\beta$ iguais a $-0,051,-0,042$ e $-0,046$, respectivamente. Em tais condições, estimam-se velocidades de convergência de $6,49 \%, 4,96 \%$ e $7,71 \%$ ao ano, nessa ordem. $A$ velocidade encontrada no período $2008-2015$ contribui menos com o processo de convergência comparativamente ao período 2000-2007. Tal fenômeno pode ser comprovado considerando a meia vida encontrada. A meia vida é uma medida de convergência indicando o tempo necessário em anos para percorrer metade da diferença entre o valor inicial da variável em estudo e aquele valor do estado estacionário.

Dada a velocidade de convergência e mantidas as condições dos períodos em análise, estima-se que serão necessários cerca de 13 e 16 anos para percorrer $50 \%$ da convergência na produtividade média da castanha de caju dos municípios cearenses nos períodos de 2000-2007 e 2008-2015, respectivamente. Assim, a convergência na taxa de produtividades dos municípios cearenses sugere que tal 
fenômeno tende a crescer mais rapidamente nas localidades menos produtivas comparativamente as demais. Nesse sentido, a diferença existente nessas taxas deverá ser gradativamente eliminada ao longo dos anos.

Ressalte-se que na presente abordagem avalia-se somente a convergência absoluta. Logo, pressupõe-se que os municípios analisados apresentam iguais níveis de rendimento médio per capita em estado estacionário, e que os possíveis diferenciais existentes nos níveis iniciais de produto médio per capita decorrem em função dos desvios de curto prazo que afetam o estoque de capital físico per capita dos municípios em relação ao seu nível em estado estacionário.

Nesse sentido, é recomendável que pesquisas futuras incorporem também elementos de políticas públicas voltadas ao segmento sócio produtivo, tais como: assistência técnica, beneficiamento e comercialização da produção, infraestrutura e reforma agrária (SILVA; NAGEM, 2015).

Por fim, aparentemente, a produtividade da castanha sofreu impacto relevante, sobretudo entre 2008 e 2015, que levou a uma queda em sua taxa de convergência. Inclusive, dividir o período de quinze anos em dois períodos (20002007 e 2008-2015) mostrou que o segundo contribui relativamente menos com o processo de convergência no setor cajucultor comparativamente ao primeiro.

Como Pedrão (2017) afirma, as perspectivas da produção rural dependem de fatores econômicos, técnicos e políticos. Assim sendo, deve-se ter em vista os possíveis efeitos negativos da crise econômica mundial iniciada em 2008 sobre o setor externo. No caso, esse evento pode ter afetado a produtividade do setor agrícola por meio do encarecimento de maquinaria, fertilizantes e outros insumos. No entanto, ressalte-se a necessidade de estimar modelos de convergência relativa para poder verificar possíveis mudanças locais ou técnica que possam ter influenciado esse processo de convergência no estado.

\section{CONSIDERAÇÕES FINAIS}

O artigo analisou a incidência de convergência na produtividade média da castanha de caju nos municípios cearenses nos períodos 2000-2007, 2008-2015 e 2000-2015. Para tanto, estimou-se um modelo de convergência considerando possíveis transbordamentos espaciais utilizando-se instrumentos de análise da econometria espacial uma vez que instrumentos tradicionais não se apresentaram adequados para modelar o problema em questão, sobretudo devido à presença de dependência espacial relevante.

Em todos os períodos analisados, foram constatados valores relevantes na dependência espacial global e local. Além disso, tal processo de dependência sugere dados mais similares entre si. Assim, municípios que apresentam alto rendimento médio no setor cajucultor parecem estar próximos de outros que também apresentam alto rendimento médio. Por sua vez, municípios que apresentam baixo rendimento médio na produtividade, geralmente, relacionam-se com outros também de baixo rendimento médio.

A partir dos modelos estimados, pode-se confirmar a hipótese de convergência na produtividade média da castanha de caju no estado do Ceará. No entanto, tal processo parece ocorrer de maneira heterogênea no período de 2000 a 2015, frente ao destaque da velocidade no período de 2000 a 2007 comparativamente ao período de 2008 a 2015. Aparentemente, a hipótese de que 0 efeito da crise econômica iniciada em 2008 tenha afetado negativamente a produtividade do setor produtor de castanha de caju não pode ser rejeitada.

Diante do exposto, deixa-se como recomendação que trabalhos futuros incorporem controles buscando capturar efeitos locais dos próprios municípios (nível 
tecnológico de plantio, colheita, escoamento do produto, estoques de capital físico e humano, fatores climáticos) que possam auxiliar no entendimento da existência do diferencial observado na produtividade no setor cajucultor cearense. Outra medida recomendável é a incorporação de variáveis capazes de representar influências do setor externo sobre a produtividade média da castanha. Para tanto, pode-se utilizar a quantidade exportada do fruto em nível municipal.

\section{REFERÊNCIAS}

ALBUQUERQUE et al. A competitividade externa da amêndoa de castanha de caju brasileira no período de 1990 a 2007. In: Eveline Babosa Silva Carvalho, Nicolino Trompieri, Jimmy Oliveira, Cleyber Nascimento e Fátima Juvenal. (Org.). Economia do Ceará em Debate 2010. Fortaleza: Imprensa do Governo do Ceará, 2011, v. 1, p. 1-25.

ALMEIDA, E. Econometria espacial aplicada. Campinas, SP: Editora Alínea, 2012.

ALMEIDA. E. S.; PEROBELLI, F. S.; FERREIRA, P. G. C. Existe convergência espacial da produtividade agrícola no Brasil? RER, Rio de Janeiro, v. 46, n. 1, p. 31$52,2008$.

ANJOS JUNIOR et al. O rendimento da castanha de caju: uma análise espacial para o estado da Paraíba. Reflexões Econômicas, v. 2, n. 2, p. 37-54, 2017.

ANSELIN, L. The Moran scatterplot as an ESDA tool to assess local instability in spatial association. In: Fisher, M, Scholten, H.J e Unwin, D W (eds). Spatial analytical perspectives in GIS. London: Taylor \& Francis, 1996, p. 111-125.

BERA, A. Spatial dependence in linear regression models with an introduction to spatial econometrics. In: Ullah A. and Giles D. E. (Ed.) Handbook of Applied Economic Statistics. New York: Marcel Dekker, 1998, p. 237-289.

Exploratory spatial data analysis and geographic information systems. In: PAINHO, M. (Ed.) New tools for spatial analysis: proceedings of the workshop. Luxemburgo: Euro Stat, 1994, p. 45-54.

. Exploring Spatial Data with GeoDa: A Workbook. Center for Spatially Integrated Social Science. 2005. Disponível em: <http://www.csiss.org/clearinghouse/GeoDa/>. Acesso em: ago. 2016.

. Interactive techniques and exploratory spatial data analysis. In: Longley, P.; Goodchild, M.; Maguire, D.; Rhind, D. Geographical information systems: Principles, Techniques, Management and Application. 2. ed. New York: John Wiley \& Sons, 1999.

. Local indicator of spatial association - LISA. Geographical Analysis, v. 27, n. 2, p. 93-115, $1995 . \quad$ Disponível em: <http://isites.harvard.edu/fs/docs/icb.topic868440.files/Anselin1995\%20LISA.pdf>. Acesso em: set. 2016.

Spatial externalities, spatial multipliers and spatial econometrics. International Regional Science Review, v. 26, n. 2, 2003. Disponível em: 
<http://web.pdx.edu/ crkl/SEAUG/papers/Anselin_IRSR_2003.pdf>. Acesso em: nov. 2016.

Spatial Econometrics: Methods and Models. Dordrecht, The Netherlands: Kluwer Academic Publishers, 1988.

BARRETO, R. C. S.; ALMEIDA, E. A contribuição da pesquisa para convergência e crescimento da renda agropecuária no Brasil. Revista de Economia e Sociologia Rural, v. 47, n. 3, p. 719-737, 2009.

BAUMOL, W. J. Productivity growth, convergency, and welfare: What the long-run show. American Economic Review, v. 76, n. 5, p. 107-285, 1986.

BRUNSDON, C.; FOTHERINGHAM, A. S.; CHARLTON, M. Geographically weighted summary statistics - a framework for localised exploratory data analysis. Computers, Environment and Urban Systems, v. 26, n. 6, p. 501-524, 2002.

CASTRO, L. S.; ALMEIDA E. S.; LIMA, J. E. A convergência espacial da produtividade de soja no Brasil: o caso das regiões Centro-Oeste e Sul. Espacios, v. 36, n. 21, 2015.

FLORAX, R. J. G. M.; FOLMER, H.; REY, S. J. Specification searches in spatial econometrics: The relevance of Hendry's methodology. Regional Science and Urban Economics, v. 33, n. 5, p. 557-79, 2003.

FREITAS, M. V.; ALMEIDA, E. Existe realmente convergência de renda entre países? Estudos Econômicos (São Paulo), v. 45, n. 2, p. 287-316, 2015.

GEARY, R. C. The contiguity ratio and statistical mapping. The Incorporated Statitician, v. 5, n. 3, p. 115-145, 1954.

GETIS, A.; ORD, J. K. The analysis of spatial association by use of distance statistics. Geographical analysis, v. 24, p. 189-206, 1992.

GUANZIROLI et al. Barreiras às novas formas de coordenação no agrossistema do caju na região nordeste, Brasil. Revista Gestão \& Produção, São Carlos, v. 17, n. 2, p. 229-244, 2010.

IBGE. Instituto Brasileiro de Geografia e Estatística. Produção Agrícola Municipal 2015, Rio de Janeiro, 2016. Disponível em: <https://sidra.ibge.gov.br/tabela/106>. Acesso em: dez. 2016.

KELEJIAN, H. H.; PRUCHA, I. R. A generalized spatial two-stage least squares procedure for estimating a spatial autoregressive model with autoregressive disturbances. J. Real State Finance Econ., Dordrecht, v. 17, n. 1, p. 99-121, 1998.

Specification and Estimation of Spatial Autoregressive Models with Autoregressive and Heteroskedastic Disturbances. Journal of Econometrics, Amsterdam, v. 157, n. 1, p. 53-67, 2010.

HAC Estimation in a Spatial Framework. Journal of Econometrics, Amsterdam, v. 140, n. 1, p. 131-154, 2007. 
A Generalized Moments Estimator for the Autoregressive Parameter in a

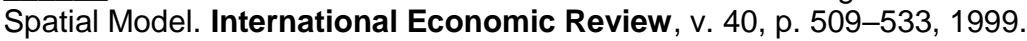

LESAGE, J. P.; PACE, R. K. Introduction to Spatial Econometrics. Chapman and Hall/CRC Press: Boca Raton, 2009.

LOPES, J. L. Avaliação do processo de convergência da produtividade da terra na agricultura brasileira no período de 1960 a 2001. Tese de Doutorado, ESALQ/USP, 2004. (mimeo).

MORAN, P. A. P. The interpretation of statistical maps. Journal of the Royal Statistical Society B, v. 10, p. 243-251, 1948.

MOURA, D.; MAGALHÃES, F. C. A castanha de caju no contexto do Programa de Aquisição de Alimentos (PAA). Revista de Política Agrícola, Brasília, DF. n. 1, jan./fev./mar. 2008. Acesso em: 6 dez. 2016.

PEDRÃO, F. C. Condições sócio estruturais da produção rural no Brasil. Extensão Rural, Santa Maria, v. 24, n. 2, p. 7-21, 2017. Disponível em: https://periodicos.ufsm.br/extensaorural/article/view/27375.

QUAH, D. T. Empirics for economic growth and convergence. European economic review, v. 40, n. 6, p. 1353-1375, 1996.

REY, J. S.; MONTOURI, B. D. US Regional income convergence: a spatial econometric perspective. Regional Studies, v. 33, n. 2, p. 143-156, 1999.

SILVA, S. P.; NAGEM, F. A. A dinâmica das políticas públicas de desenvolvimento rural e sua incidência territorial: uma análise do Pronaf no Território Vale do Murici/MG. Extensão Rural, Santa Maria, v. 22, n. 2, p. 60-78, 2015. Disponível em: https://periodicos.ufsm.br/extensaorural/article/view/13407. 
Apêndice A - Teste de convergência do rendimento médio da castanha de caju no estado do Ceará

Tabela 5 - Teste de $\sigma$-convergência do rendimento médio da castanha de caju no estado do Ceará - anos 2000, 2008 e 2015

\begin{tabular}{l|l|l|l|l|l|l}
\hline & 2000 & 2008 & 2015 & $2000-2008$ & $2008-2015$ & $2000-2015$ \\
\hline C.V. & 0,54 & 0,37 & 0,80 & $-0,17$ & 0,43 & 0,26 \\
\hline
\end{tabular}

Fonte: elaboração dos autores a partir de dados da pesquisa.

Nota: C.V. é o coeficiente de variação. 